\title{
Testing the reality of the quantum state
}

To the editor - Pusey, Barrett and Rudolph have proved a mathematically neat theorem that assesses the reality of the quantum state (M. F. Pusey, J. Barrett and T. Rudolph, Nature Phys. 8, 475-478; 2012). They proposed a test such that if any pair of quantum states could pass it, then for small deviations in the probabilities of measurement outcomes, $\varepsilon$, from the predicted quantum probabilities, one can conclude that the physical state $\lambda$ "is normally closely associated with only one of the two quantum states". Although the mathematics of their theorem is correct, the physical interpretation is incomplete.

Here, I present an argument that greatly limits the conclusion that can be drawn from even a successful test. Specifically, I show that the physical state can be associated with several quantum states and, thus, the reality of quantum states cannot be deduced.

The procedure for implementing Pusey and colleagues' circuit test is as follows. Pick two distinct quantum states $\left|\psi_{0}\right\rangle,\left|\psi_{1}\right\rangle$ that determine the angle $\theta$ in the equations:

$$
\begin{aligned}
& \left|\psi_{0}\right\rangle=\cos (\theta / 2)|0\rangle+\sin (\theta / 2)|1\rangle \\
& \left|\psi_{1}\right\rangle=\cos (\theta / 2)|0\rangle-\sin (\theta / 2)|1\rangle
\end{aligned}
$$

where $|0\rangle$ and $|1\rangle$ constitute a basis for the Hilbert space, which is treated as the computational basis. Then, find the minimum integer $n$ that satisfies $n \geq \ln 2 / \ln (1+\tan (\theta / 2))$ and label this integer $n(\theta)$. Now, construct the circuit test of Pusey et al. for $n(\theta)$, perform the experiment and find $\varepsilon$. The theorem states that the total variation distance, $D\left(\mu_{0}, \mu_{1}\right)$, between the distributions $\mu_{0}$ and $\mu_{1}$ of the physical states satisfies the inequality $D\left(\mu_{0}, \mu_{1}\right) \geq D_{\mathrm{lb}}(\varepsilon, n)$, where $D_{\mathrm{lb}}(\varepsilon, n)=1-2 \sqrt[n]{\varepsilon}$, is the lower bound distance. Pusey et al. conclude that "for small $\varepsilon, D\left(\mu_{0}, \mu_{1}\right)$ is close to 1 . Hence, a successful experiment would show that $\lambda$ is normally closely associated with only one of the two quantum states".

The conclusion would be accurate if there was no minimum for $\varepsilon$. However, if, due to practical limitations, there is a minimum for $\varepsilon\left(\varepsilon_{\min }\right)$ that cannot be passed for any $\theta$, then the test would not be very informative.

Here, I assume that at "any epoch in the history" there exists an $\varepsilon_{\min }$. Note that for small $\theta$, large $n(\theta)$ is required. As $n(\theta)$ gets larger and larger, $D_{\mathrm{lb}}(\varepsilon, n(\theta))$ becomes smaller and smaller for $\varepsilon \geq \varepsilon_{\text {min }}$. In fact, if $\theta$ becomes sufficiently small, $D_{\mathrm{lb}}(\varepsilon, n(\theta))$ becomes negative.

Let us define two regions for $\theta$ : a far region in which $D_{\mathrm{lb}}\left(\varepsilon_{\min }, n(\theta)\right)>1 / 2$ and a near region where $D_{\mathrm{lb}}\left(\varepsilon_{\min }, n(\theta)\right) \leq 1 / 2$. Neither of these regions is empty. In fact, it can easily be checked that every $\theta$ satisfying

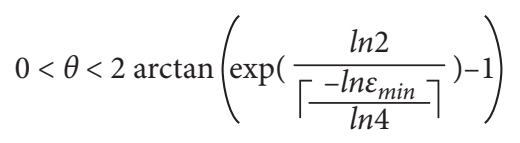

belongs to the near region, where $\lceil\cdot\rceil$ is the ceiling function.

Now, note that for any $\varepsilon \geq \varepsilon_{\min }$, $D_{\mathrm{lb}}(\varepsilon, n) \leq D_{\mathrm{lb}}\left(\varepsilon_{\min }, n\right)$. Therefore, if a pair of quantum states belongs to the near region, $D_{\mathrm{lb}}\left(\varepsilon_{\min }, n(\theta)\right) \leq 1 / 2$. This pair can pass Pusey and colleagues' circuit test for some small value of $\varepsilon$. The fundamental equation of their paper, eq. (7), states, for this pair, that $D\left(\mu_{0}, \mu_{1}\right) \geq D_{\mathrm{lb}}(\varepsilon, n(\theta))$. As $D_{\mathrm{lb}}(\varepsilon, n(\theta)) \leq 1 / 2$, we can conclude that for the states in the near region, the most restrictive constraint Pusey and colleagues' theorem can impose is just $D\left(\mu_{0}, \mu_{1}\right) \geq 1 / 2$.

As this is the only constraint, then a physical model can easily be constructed in which the area of the overlapping region between $\mu_{0}$ and $\mu_{1}$ is at least $25 \%$ for the states in the near region (for example, consider a square overlap region for $\mu_{0}$ and $\mu_{1}$ ). This means that the states of a pair are not in one-to-one correspondence with physical states and hence they are not ontic.

In fact, for any fixed $\left|\psi_{0}\right\rangle$ we can write $\left|\psi_{1}(\theta)\right\rangle$ states as

$$
\left|\psi_{1}(\theta)\right\rangle=\cos (\theta)\left|\psi_{0}\right\rangle+\sin (\theta)\left|\psi_{0}^{\perp}\right\rangle
$$

where $\left|\psi_{0}^{\perp}\right\rangle=\sin (\theta / 2)|0\rangle-\cos (\theta / 2)|1\rangle$ is orthogonal to $\left|\psi_{0}\right\rangle$. Now it can be seen that there are many, mathematically infinite, numbers of $\left|\psi_{1}(\theta)\right\rangle$ in the near neighbourhood (region) of $\left|\psi_{0}\right\rangle$.

All $\left|\psi_{1}(\theta)\right\rangle$ can, in principle, pass the circuit test and satisfy the inequality $D\left(\mu_{0}, \mu_{1}\right) \geq D_{\mathrm{lb}}(\varepsilon(\theta), n(\theta))$, and for many $\left|\psi_{1}(\theta)\right\rangle$, from the far region, it might be found that $D_{\mathrm{lb}}(\varepsilon(\theta), n(\theta))$ is close to 1 . However, this does not mean that there is no $\left|\psi_{1}(\theta)\right\rangle$ whose distribution of physical states overlaps significantly with that of $\left|\psi_{0}\right\rangle$. In fact, the entire near region of $\left|\psi_{0}\right\rangle$ is filled with such quantum states.

This, in effect, means that any $\lambda$ in support of the distribution of $\left|\psi_{0}\right\rangle$ corresponds to $\left|\psi_{0}\right\rangle$ and, at the same time, to many $\left|\psi_{1}(\theta)\right\rangle$ from the near neighbourhood of $\left|\psi_{0}\right\rangle$. This strongly disagrees with the conclusion of Pusey et al., which was based on the results of a successful experiment.

In other words, even if a successful Pusey et al. experiment for a pair of quantum states $\left|\psi_{0}\right\rangle,\left|\psi_{1}\right\rangle$ can be made, and it is found that $D\left(\mu_{0}, \mu_{1}\right)$ is close to 1 , it cannot be inferred that the physical states are in one-to-one correspondence with the quantum states. The reason is that, as shown here, there are many quantum states in the near neighbourhood of each $\left|\psi_{0}\right\rangle,\left|\psi_{1}\right\rangle$ that have a significant amount of physical states in common with $\left|\psi_{0}\right\rangle$, $\left|\psi_{1}\right\rangle$, and Pusey and colleagues' proposal is unable to say anything useful about them. This shows that their proposal falls short of being an exclusive test and the work needs extending.

\section{Acknowledgements}

I would like to thank A. J. Leggett for useful comments on an earlier version of the manuscript. This work was supported by the MacArthur Professorship endowed by the John D. and Catherine T. MacArthur Foundation at the University of Illinois.

Seyyed M. H. Halataei

University of Illinois at Urbana-Champaign, 1110 W Green Street, Urbana,

Illinois 61801, USA.

e-mail:halatae1@illinois.edu
Pusey et al. reply - We did not claim in our original Article (M. F. Pusey, J. Barrett \& T. Rudolph, Nature Phys. 8, 475-478; 2012) that all models of the form considered in which the quantum state is not real can be ruled out by a single experiment. Rather, we claimed that for any such model there exists an experiment that is, in principle, feasible and would rule it out. The argument presented by Halataei (S. M. H. Halataei Nature Phys. 10, 174; 2014) "limits the conclusion one can draw from even a successful test" no further than this. We agree with the mathematical claim of the comment: for a fixed value of $\varepsilon$, the experimental noise, an infinite number of quantum state pairs can always be found that are sufficiently close together that an 